\title{
H.pylori and Pepsin as a Putative Risk Factors in Laryngeal Cancer Patients
}

Original Article

\author{
Heba Mahmoud ${ }^{1}$, Ahmed Mohamed Kamel', Amr Hamed Mohamed', Mohamed \\ Shehata Taha', Anas Askoura ${ }^{1}$, Azza Omran', Basma S. Mahmoud ${ }^{3}$ and Soha \\ Aboelela $^{3}$
}

${ }^{1}$ Department of Ear, Nose and Throat, ${ }^{3}$ Department of Clinical Pathology, Faculty of Medicine, Ain-Shams University, ${ }^{2}$ Department of Clinical Pathology, Al-Mataria Teaching Hospital, Cairo, Egypt.

\begin{abstract}
Background: Laryngeal cancer constitutes $26-30 \%$ of all head and neck malignant tumors. Chronic stimulation by acid reflux may lead to a malignant change in the laryngopharyngeal mucosa. Pepsin assay could be a rapid, sensitive, and specific diagnostic marker of extra-esophageal reflux (EES). Helicobacter pylori is involved in the pathogenesis of peptic ulcer, and gastric carcinoma with growing interest in the presence of Helicobacter pylori in the upper aerodigestive tract. Aim: The aim of the study is to confirm the role of H.pylori and pepsin as risk factors in laryngeal cancer patients. Patients and Methods: Seventy-five patients were enrolled in this study, and divided into 3 groups; Group (A) 32 patients with suspected laryngeal malignancy. Group (B) 20 patients with benign laryngeal lesions. Group (C) 22 patients free from laryngeal disease, scheduled for non-laryngeal surgery. All patients were subjected to laryngo-pharyngeal lavage (LPL) using sterile water, before starting the surgical procedure and was analyzed for detection of Pepsin an H. Pylori, also culture for H. Pylori was done.

Results: Strong positive association exist between H. pylori and Pepsin and occurrence of laryngeal carcinoma, while there was no statistically significant relationship with occurrence of a benign laryngeal lesions.

Conclusion: GER is a risk factor or co-carcinogen in the pathogenesis of laryngeal carcinoma and not considered as a risk factor for benign laryngeal lesions. H. pylori culture and Pepsin assay in LPL can be used as non-invasive tool for detection of GER.
\end{abstract}

Key Words: Bamford- Cancer larynx, H. pylori, pepsin.

Received: 24 September 2020, Accepted: 12 November 2020

Corresponding Author: Anas Askoura, MD, Department of Ear Nose and Throat, Faculty of Medicine, Ain-Shams University, Egypt, Tel.: +201113110666, E-mail: anasaskoura@gmail.com

ISSN: 2090-0740, 2021 Vol.22

\section{INTRODUCTION}

The 5-year survival of patients with head and neck squamous cell carcinoma (HNSCC) has not changed dramatically over the last decades despite the use of various diagnostic and therapeutic modalities ${ }^{[1]}$. Thus, new approaches remain necessary to prevent cancer, improve treatment and reduce recurrence. Laryngeal cancer constitutes $26-30 \%$ of all head and neck malignant tumors $^{[2]}$. It is common in men and it is distributed between ages 40 and 70 years old. Smoking, alcohol, human papilloma virus and radiation are well-known etiologic factors of laryngeal cancer. Other reported risk factors include, genetics, carotene deficiency ${ }^{[3]}$ and occupational exposure $^{[4,5]}$.

The hypothesis that gastric reflux plays a role in the development of laryngeal squamous cell carcinoma, was first suggested by Gabriel and Jones ${ }^{[6]}$ in 1960. Other study concluded that, chronic stimulation by acid reflux may lead to a malignant change in the laryngopharyngeal mucosa ${ }^{[7]}$. A high frequency of acid reflux has been reported Since the mid-80's in laryngeal cancer patients who do not drink alcohol or smoke cigarettes, this lead to an increased interest in the relationship between laryngeal cancer and acid reflux ${ }^{[8,9,10]}$.

Despite the recognition that gastroesophageal reflux (GER) can provoke laryngeal cancer, the diagnosis of laryngopharyngeal reflux (LPR) remains a very difficult task. Initially, patients with laryngeal symptoms undergo laryngoscopy and chest X-ray to rule out malignancies. Once cancer is excluded, a diagnosis of LPR is suspected when the following laryngoscopic findings are seen: edema, erythema, ventricular obliteration, pseudosulcus and postcricoid hyperplasia. However, these laryngoscopic findings 
are also common in healthy volunteers, and this largely limits their diagnostic value ${ }^{[11]}$. Moreover, there are several controversies regarding how to confirm LPR. Upper gastrointestinal endoscopy has been demonstrated to have low sensitivity ${ }^{[12,13]}$, the proton pump inhibitor test has been shown to have low specificity ${ }^{[14]}$, and radiologic studies have limited sensitivity and specificity ${ }^{[12]}$. Nowadays it was found that multichannel intraluminal impedance and $\mathrm{pH}$ monitoring (MII-pH) is able to detect not only acid but also non-acid reflux and proximal migration of the refluxate $^{[15,17]}$. However, there is still a great debate of controversy surrounding the significance of LPR role.

Pepsin exists in the form of pepsinogen, which is secreted mainly by stomach mucosa cells. Pepsinogen exerts its biological activity when converted to pepsin if the $\mathrm{pH}$ is decreased. Under normal conditions Pepsin is not detected in the laryngopharyngeal lavage (LPL). When reflux occurs into the throat, laryngeal mucosa cells may be damaged ${ }^{[18]}$. Recent studies have shown that pepsin can be used as a diagnostic marker of $\operatorname{LPR}^{[19,20]}$.

Helicobacter pylori is a gram-negative bacterium involved in the pathogenesis of peptic ulcer, and gastric carcinoma. The ecological niche of $\mathrm{H}$. pylori is the human stomach, where it establishes long-term colonization of the gastric mucosa ${ }^{[21]}$. There is growing interest in the presence of Helicobacter pylori in the upper aerodigestive tract ${ }^{[22]}$. The aim of this study is to investigate the role of H.pylori and pepsin as risk factors in laryngeal cancer patients.

\section{PATIENTS AND METHODS:}

This was a prospective study carried out between January 2018 and January 2020, after approval of the Institutional Review Board of Ain Shams University Hospitals. An informed consent was signed by all participants. Seventy five patients were enrolled in this study, and divided into 3 groups (A, B and C). All participants subjected to detailed history taking including history of reflux and full ENT examination. Group (A) 32 patients with suspected laryngeal malignancy. Group (B) 21 patients with benign laryngeal lesions. Group (C) 22 patients free from laryngeal disease, scheduled for nonlaryngeal surgery (10 for septoplasty, 6 for tonsillectomy and 4 for myringoplasty) were included as control.

Prior to surgery routine laboratory investigations, chest $\mathrm{X}$-ray and laryngeal photography were done. Contrast enhanced CT, only for laryngeal cancer patients. Group (A) underwent microlaryngosurgery for lesion mapping and biopsy taking. While excisional biopsy was done for Group (B). All biopsies were sent for histopathological examination. Group (A) patients with proven laryngeal malignancy underwent total, partial laryngectomy and neck dissection according to the staging of the lesions and extent of the pathology. Post-resection histopathology reports and disease staging were recorded.

Exclusion criteria were as follows: Previous surgery in the upper aerodigestive tract, eating disorders with vomiting, pregnancy and/or breastfeeding, use of aspirin, non-steroidal anti-inflammatory drugs, treatment for reflux either medical or surgical and underlying psychiatric illness.

All patients were subjected to laryngo-pharyngeal lavage (LPL) using sterile water, before starting the surgical procedure under general anesthesia. Samples were placed on ice and immediately transferred to the laboratory, where it was stored at $-80^{\circ} \mathrm{C}$ until analyzed.

\section{The stored samples were analyzed with:}

1- Campylobacter-like organism "CLO" (rapid urease assay) kit (Ballard medical product, Draper, Utah, USA) which is based on the fact that the LPL can be inoculated into a gel (medium containing urea and phenol red, a dye that turns pink at $\mathrm{pH} 6.0$ or higher). Thus the PH rises above 6.0 when H.pylori metabolizes urea to ammonia via urease activity. The sample that did not change in color and remained yellow was considered negative, while sample that turns orange or red recorded as H.pylori positive.

2- Enzyme-linked immunosorbent assay (ELISA) kit was used for detection of pepsin (Fujian Blueprint Technology Company, FuZhou, China). This assay was carried out according to the manufacturer's instructions. Absorbance values of the samples at $450 \mathrm{~nm}$ were determined using a microplate reader (BioTek Instruments, Inc., Winooski, VT). Results were classified as positive if pepsin was detected in LPL.

3- Culture of $\mathrm{H}$. pylori on Columbia agar supplemented with lysed horse blood and Skirrow's supplement (containing vancomycin, trimethoprim, polymyxin B and amphotericin B) (Oxid-Unipath, limited, Pasingstoke, and Hampshire, England). The plates were incubated under microaerobic conditions $\left(90 \% \mathrm{CO}_{2}, 5 \%\right.$ $\mathrm{O}_{2}, 5 \% \mathrm{~N}_{2}$ ) at $37{ }^{\circ} \mathrm{C}$ for 4 to 7 days. Suspected colonies of H.pylori (small, circular, convex, translucent) were tested by gram stain, and catalase activities.

\section{STATISTICAL METHODS:}

Data were analyzed using IBMC SPSSC Statistics version 22 (IBMC Corp., Armonk, NY). Numerical variables were presented as mean and standard deviation and inter-group differences were compared using oneway analysis of variance (ANOVA) with application of the Tukey test for post hoc comparisons if needed. 
Categorical variables were presented as ratio or as number and percentage and differences were compared using the Pearson chi-squared test. Associations between nominal variables on two levels were tested using the phi coefficient of association $(\varphi)$. Multinomial logistic regression analysis was used to examine the relation between CLO test, $\mathrm{H}$. pylori or positive pepsin test and occurrence of laryngeal lesions. Two-sided p-values $<0.05$ are considered statistically significant.

\section{RESULTS:}

Patients were divided into three groups. Group (A) 32 patients with suspected laryngeal malignancy. Their mean age was $57.0 \pm 10$, and there were $30(93.75 \%)$ males and $2(6.25 \%)$ females. $24(75.0 \%)$ of them were smoker. Group (B) 21 patients with benign laryngeal lesions, their mean age was $46 \pm 10$, and there were $11(52.4 \%)$ males and $10(47.6 \%)$ females. $11(52.4 \%)$ of them were smoker. Group (C) 22 patients free from laryngeal disease, scheduled for non-laryngeal surgery (10 for septoplasty, 6 for tonsillectomy and 4 for myringoplasty) were included as control. Their mean age was $47 \pm 9$, and there were 16 $(72.7 \%)$ males and $6(27.3 \%)$ females. $14(63.4 \%)$ of them were smoker.

Clinical and histopathological characteristics of group (A) And (B) are reveled in (Table 1,2). Results of CLO test, H. pylori culture and pepsin test in the three studied groups are presented in (Table 3 ) and (Figure 1). There was a relatively strong positive association between CLO, $\mathrm{H}$. pylori or Pepsin and occurrence of laryngeal carcinoma (phi coefficient, $\varphi=0.61,95 \% \mathrm{CI}=0.44$ to 0.79 , $p$-value $<0.001$ ) (Table 4).

The sensitivity, specificity, efficiency, and prevalence of CLO, H.pylori or pepsin for prediction of laryngeal malignancy in patients presenting with a laryngeal lesions were $(88 \%, 71 \%, 81 \%$ and $60 \%$ respectively). After adjustment for patient' age, sex and smoking status, there was no statistically significant relation between CLO (or H. pylori or pepsin) and occurrence of a benign laryngeal lesion (odds ratio $=1.3,96 \% \mathrm{CI}=0.3$ to 5.3 , $p$-value $=0.753$ ). However, the relation between CLO (or H. pylori or pepsin) and malignant laryngeal lesion was statistically significant (odds ratio $=31.0,96 \% \mathrm{CI}=5.4$ to 178.4.4, p-value $<0.001$ ). (Table 5).
Table 1: Clinical and histopathological characteristics of malignant laryngeal lesions

\begin{tabular}{lc}
\hline TNM stage, histopathological type & Number of patients (\%) \\
\hline T stage & $6(18.8 \%)$ \\
T1 & $1(3.1 \%)$ \\
T2 & $14(43.8 \%)$ \\
T3 & $11(34.4 \%)$ \\
T4 & \\
N stage & $16(50.0 \%)$ \\
N0 & $9(28.1 \%)$ \\
N1 & $6(18.8 \%)$ \\
N2 & $1(3.1 \%)$ \\
N3 & \\
M stage & $32(100.0 \%)$ \\
M0 & $0(0.0 \%)$ \\
M1 & \\
Clinical stage & $6(18.8 \%)$ \\
Stage I & $0(0.0 \%)$ \\
Stage II & $10(31.3 \%)$ \\
Stage III & $16(50.0 \%)$ \\
Stage IV & $1(3.1 \%)$ \\
Histopathological type & $11(34.4 \%)$ \\
Carcinoma in-situ & $16(50.0 \%)$ \\
Well differentiated carcinoma & $4(12.5 \%)$ \\
Moderately differentiated & \\
carcinoma & \\
\hline
\end{tabular}

Table 2: Clinical and histopathological characteristics of benign laryngeal lesions

Site and histopathological type $\quad$ Number of patients $(\%)$

Site

Supraglottic

$0(0.0 \%)$

Glottic

$21(100 \%)$

Transglottic

$0(0.0 \%)$

Histopathological type

Vocal fold polyp

$8(38.1 \%)$

Postglottic granuloma

$4(19.0 \%)$

Reinks edema

$3(14.3 \%)$

Laryngeal papillomatosis

$4(19.0 \%)$

Vocal fold keratosis

$2(9.5 \%)$ 
Table 3: Results of CLO culture, H. pylori culture and pepsin test in the three study groups

\begin{tabular}{lccccc}
\hline Test & Result & Cancer larynx $(\mathrm{n}=32)$ & Benign laryngeal lesions $(\mathrm{n}=21)$ & No laryngeal lesion $(\mathrm{n}=22)$ & $p$-Value ${ }^{+}$ \\
\hline \multirow{2}{*}{ CLO } & Negative & $4(12.5 \%)$ & $15(71.4 \%)$ & $17(77.3 \%)$ & $<0.001$ \\
& Positive & $28(87.5 \%)$ & $6(28.6 \%)$ & $5(22.7 \%)$ & \\
\multirow{2}{*}{ H. pylori culture } & Negative & $4(12.5 \%)$ & $15(71.4 \%)$ & $17(77.3 \%)$ & $<0.001$ \\
& Positive & $28(87.5 \%)$ & $6(28.6 \%)$ & $5(22.7 \%)$ & \\
\multirow{2}{*}{ Pepsin } & Negative & $4(12.5 \%)$ & $15(71.4 \%)$ & $17(77.3 \%)$ & $<0.001$ \\
& Positive & $28(87.5 \%)$ & $6(28.6 \%)$ & $5(22.7 \%)$ & \\
\hline
\end{tabular}

Data are number (percentage).

$\uparrow$. Pearson chi-squared test.

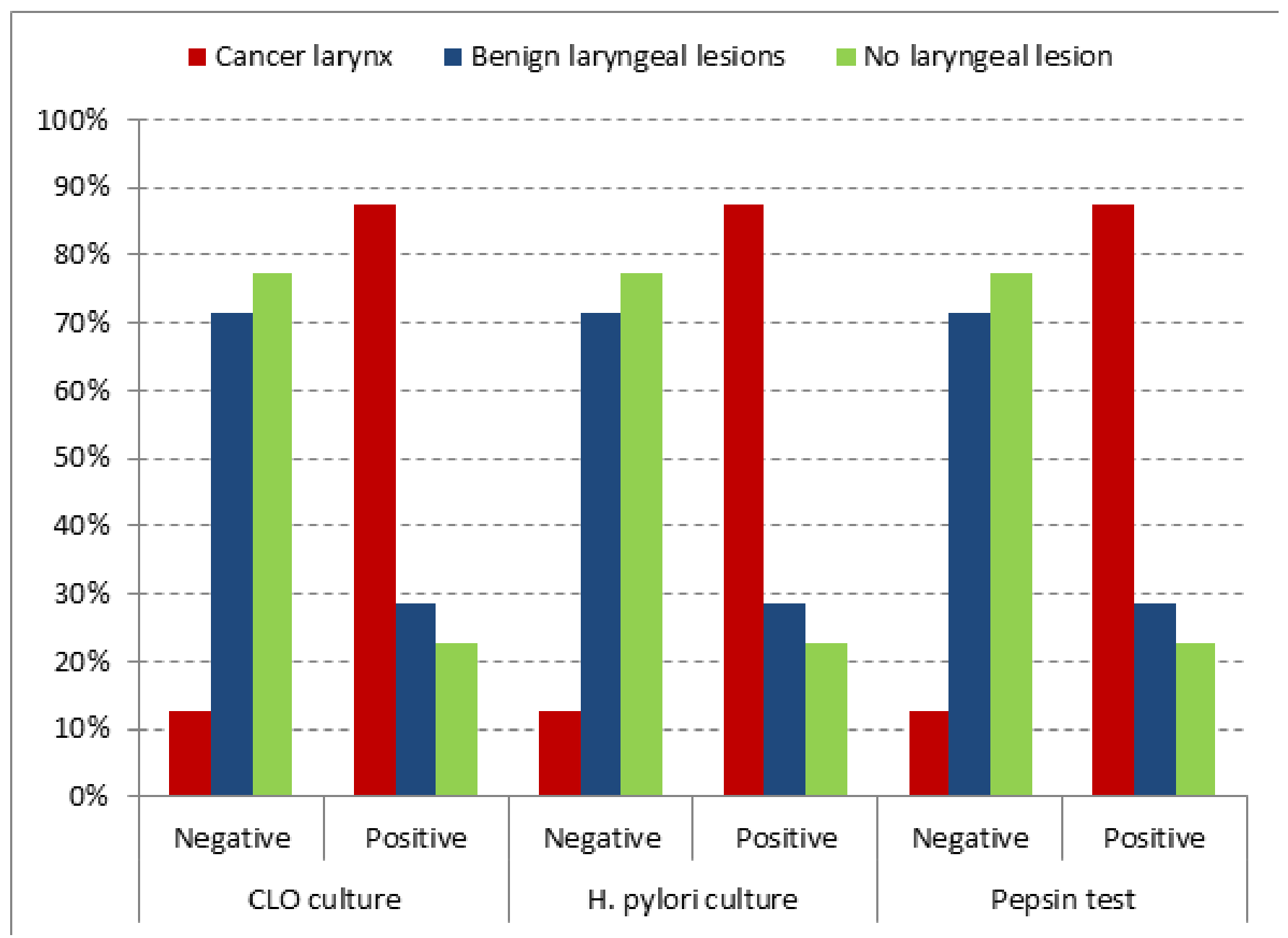

Fig. 1: Results of CLO culture, H. pylori culture and pepsin test in the three study groups. 
Table 4: Association between CLO, H. pylori or pepsin and the occurrence of laryngeal cancer

\begin{tabular}{ccccccc}
\hline & \multicolumn{3}{c}{ Test (CLO, H. pylori or Pepsin) } \\
\hline \multirow{3}{*}{ Criterion } & Positive & Negative & Columns total & \multicolumn{2}{c}{ Measure of association } \\
& Malignant laryngeal lesion & 28 & 4 & 32 & Phi $(\varphi)$ & 0.61 \\
& No or benign laryngeal lesion & 11 & 32 & 43 & $95 \%$ CI & 0.44 to 0.79 \\
& Rows total & 39 & 36 & 75 & $p$-Value & $<0.001$ \\
\hline
\end{tabular}

Data are counts.

Table 5: Multinomial logistic regression for the relation between CLO, H. pylori or pepsin and occurrence of benign or malignant laryngeal lesion as adjusted for patients' age and sex

\begin{tabular}{lllllllll}
\hline & & & & & & & \multicolumn{2}{c}{$95 \%$ CI for Exp(B) } \\
\hline Outcome $^{*}$ & Independent variable & $\mathrm{B}$ & $\mathrm{SE}$ & Wald (df1) & $p$-Value & Exp(B) & Lower Bound & Upper Bound \\
Benign & Intercept & 0.674 & 1.552 & 0.189 & 0.664 & & & 1.062 \\
laryngeal & Age (years) & -0.006 & 0.033 & 0.029 & 0.865 & 0.994 & 0.931 & 2.190 \\
lesion & Male sex $\dagger$ & -0.962 & 0.891 & 1.166 & 0.280 & 0.382 & 0.067 & 6.524 \\
& Smoking $\$$ & 0.165 & 0.872 & 0.036 & 0.850 & 1.180 & 0.213 & 5.251 \\
& Positive CLO (or H. pylori or pepsin) $\S$ & 0.207 & 0.740 & 0.078 & 0.780 & 1.230 & 0.288 & \\
\hline Malignant & Intercept & -9.051 & 3.138 & 8.318 & 0.004 & & & 1.231 \\
laryngeal & Age (years) & 0.110 & 0.050 & 4.915 & 0.027 & 1.117 & 1.013 & 275.430 \\
lesion & Male sex $\dagger$ & 3.028 & 1.322 & 5.249 & 0.022 & 20.654 & 1.549 & 1.975 \\
& Smoking $\dagger$ & -1.250 & 0.985 & 1.611 & 0.204 & 0.287 & 0.042 & 178.359 \\
\hline
\end{tabular}

$95 \% \mathrm{CI}=95 \%$ confidence interval, $\mathrm{B}=$ regression coefficient, $\mathrm{df}=$ degree of freedom, $\operatorname{Exp}(\mathrm{B})=$ odds ratio, $\mathrm{SE}=$ standard error, $\mathrm{Wald}=$ Wald chi-squared statistic.

*. The reference category is: No laryngeal lesion.

$\dagger$. The reference category is: Female sex.

\$. The reference category is: No smoking.

$\S$. The reference category is: Negative CLO (Negative H. pylori or Negative pepsin, respectively)

\section{DISCUSSION}

An important emerging risk factor for laryngeal cancer is GER. Many studies evaluating the incidence of laryngeal cancer, found that the age-adjusted incidence increased during the last decades ${ }^{[23,24,25]}$, despite decreasing numbers of people who smoke ${ }^{[26]}$. Such observations, along with the high reported prevalence of GER in patients with laryngeal carcinoma $^{[10,27,37]}$ suggested that GER may be an important risk factor for laryngeal carcinoma. In this study, we evaluated the role of $\mathrm{H}$. pylori and pepsin as risk factors in laryngeal cancer patients.

In the present study, 75 patients were included and divided into 3 groups. Group (A) 32 patients with laryngeal malignancy. Group (B) 21 patients with benign laryngeal lesions. Group (C) 22 patients free from laryngeal disease, scheduled for non-laryngeal surgery.

Results of CLO test in group A,B,C were (positive results in $87.5 \%, 28.6 \%$ and $22.7 \%$ respectively) and were negative in $(12.5 \%, 71.4 \%$ and $77.3 \%$
respectively).H. pylori culture results were positive in $(87.5 \%, 28.6 \%$ and $22.7 \%$ respectively), and were negative in $(12.5 \%, 71.4 \%$ and $77.3 \%$ respectively).

Pepsin assay results were (positive results in $87.5 \%$, $28.6 \%$ and $22.7 \%$ respectively) and were negative in $(12.5 \%, 71.4 \%$ and $77.3 \%$ respectively).

There was a relatively strong positive association between CLO, H. pylori and Pepsin with occurrence of laryngeal carcinoma (phi coefficient, $\varphi=0.61,95 \%$ $\mathrm{CI}=0.44$ to 0.79, p-value $<0.001)$ (Table 4$)$. The sensitivity, specificity, efficiency, and prevalence of CLO, H.pylori and pepsin for prediction of laryngeal malignancy in patients presenting with a laryngeal lesions were $(88 \%, 71 \%, 81 \%$ and $60 \%$ respectively). After adjustment for patient' age, sex and smoking status, there was no statistically significant relation between CLO, H. pylori and pepsin and occurrence of a benign laryngeal lesion (odds ratio $=1.3,96 \%$ $\mathrm{CI}=0.3$ to $5.3, p$-value $=0.753$ ). However, the relation between CLO, H. pylori and pepsin with malignant laryngeal lesion was statistically significant (odds ratio $=31.0,96 \% \mathrm{CI}=5.4$ to 178.4 .4 , $p$-value $<0.001)$. 
These results showed that GER may play a role in the development of laryngeal cancer by increasing the risk of cancer irrespective of other established risk factors as smoking, the phenomenon of independent causation or through enhances the carcinogenic potential of this factor by synergistic mechanisms, the phenomenon of co-carcinogenesis.

Many studies suggested an independent role for GER in laryngeal carcinogenesis $\left.{ }^{[29,} 39,32,37\right]$. Mercante et al., compared the incidence of GER in 274 patients with malignant neoplasm of the oral cavity, of the pharynx and of the larynx, examined by esophagogastro-duodenoscopy (EGD) with 636 controls in a retrospective study. The GER prevalence in this nonsmoking cancer subgroup was $21 \%$, significantly higher than the $5 \%$ found in the nonsmoking controls.

In a study carried out by Bacciu et al. (38) based on laryngeal cancer patients with no history of smoking or drinking and a normal control group consisting of individuals with no abnormal laryngeal findings, GER was noticed in $27.7 \%$ in the laryngeal cancer group and $4.8 \%$ in the control group.

Morrison et al[29] found that $48 \%$ of patients in the nonsmoking cancer group in his study had experienced reflux suggesting that GER may be a risk factor for laryngeal cancer. Similarly, Ward and Hanson ${ }^{[39]}$, published a retrospective series of 138 cancer patients. $12 \%$ of patients in their series were nonsmokers and nonalcoholic with moderate to severe GER, again suggesting that GER is an independent risk factor in development of laryngeal carcinoma.

Moreover, GER may act as co-carcinogen by enhancing the carcinogenic effects of smoking and alcohol. The cocarcinogenic potential of GER seems plausible given the injurious nature of refluxate to the larynx ${ }^{[28,31,40]}$. El-Serag et $a l^{[10]}$ showed that the GER patients were 2.31 times more likely to develop cancer; smoking increased the risk by 2.60 times, but in the presence of both smoking and GER, the risk increased by 2.79 times. Silent GER was evaluated in many studies using dual-probe $\mathrm{pH}$ monitoring in patients with laryngopharyngeal squamous cell carcinoma. GERD was evident in cancer patients compared to control groups ${ }^{[27,33,34,35,36]}$. From these studies, it might be easy to conclude that GERD has causal role in carcinogenesis given its high prevalence in laryngeal cancer patients.

Reflux has been documented by detection of pepsin in the trachea, lung, sinus, middle ear, combined sputum and saliva, and breath condensate ${ }^{[41]}$. In this regard, two review articles have highlighted that pepsin assay is a rapid, sensitive, and specific tool for correlation of reflux with airway disease and is a reliable diagnostic marker of extra-esophageal reflux (EES) which means that EER can now be detected by the non-invasive identification of pepsin in saliva ${ }^{[24,43]}$.

Another study by Bardhan et $a l^{[43]}$ also showed that higher concentrations of pepsin and frequency of exposure through an increased number of reflux events were more likely to result in injury of the susceptible laryngopharynx. Furthermore, the severity of reflux was related to salivary pepsin, with sever laryngopharyngeal symptoms in subjects who had salivary pepsin compared to those who did not ${ }^{[44]}$.

On the other hand, some studies found that the strength of association between GER and laryngeal cancer is uncertain. Geterude et $a l^{[45]}$ compared the occurrence and frequency of reflux in 40 consecutive cancer patients and 40 healthy volunteers. The authors reported that the number of reflux episodes and fraction of time of the $\mathrm{pH}$ less than 4 at both the proximal and distal esophageal probes were not significantly different between the patients and controls. Chen et $a l^{[46]}$ conclude in their study that, the prevalence of GER in cancer and noncancer groups were not statistically significant. Ozlugedik et al. ${ }^{[47]}$ reported a $62 \%$ occurrence rate of LPR and a $45 \%$ occurrence rate of pathologic GER in laryngeal cancer patients, although there was no significant difference when compared to the control group.

Lundell et al. ${ }^{[48]}$ showed that acid is an uncommon cause of laryngopharyngeal symptoms. A similar finding was demonstrated in study by Ang et al. ${ }^{[49]}$ suggesting that acid and non-acid refluxes do not play different roles in the genesis of extra-esophageal symptoms.

Our study showed that no statistically significant relation between $\mathrm{CLO}, \mathrm{H}$. pylori and pepsin and occurrence of a benign laryngeal lesion (odds ratio $=1.3,96 \% \mathrm{CI}=0.3$ to $5.3, p$-value $=0.753)$. This was in accordance with, Yadlapati et al ${ }^{[50]}$ who found that oropharyngeal $\mathrm{pH}$ testing and salivary pepsin analysis could not distinguish between healthy volunteers and individuals with a combination of laryngeal benign lesion. However Beltesis et al, in their study showed that $75 \%$ of patients with a VFP had laryngopharyngeal reflux (LPR), and this value is greater than that among patients with normal laryngoscopy findings ${ }^{[51]}$. Koufman et al., concluded in their study that, the incidence of LPR was significantly higher in the VFP group than in the control group ${ }^{[27]}$. 


\section{CONCLUSION}

There was a relatively strong positive association between $\mathrm{H}$. pylori and pepsin with occurrence of laryngeal carcinoma which suggest the role of GER as a risk factor or co-carcinogen in the pathogenesis of laryngeal carcinoma. On the other hand GER is not a risk factor for benign laryngeal lesions. H. pylori culture and Pepsin assay in LPL can be used as noninvasive tool for detection of GER.

\section{CONFLICT OF INTEREST}

There are no conflicts of interest.

\section{REFERENCES}

1. National Cancer Institute. Surveillance epidemiology and end results. (accessed on 21 May 2013). Available online: http://seer.cancer.gov/statfacts/html/oralcav. $\mathrm{html} /$ )

2. Muir C, Weiland L. Upper aerodigestive tract cancers. Cancer. 1995 Jan 1;75(1 Suppl):147-53.

3. Fontham ET. Protective dietary factors and lung cancer. Int J Epidemiol 1990;19: S32 - S42.

4. Brown LM, Mason TJ, Pickle LW, et al. Occupational risk factors for laryngeal cancer on the Texas Gulf Coast. Cancer Res 1988;48:1960- 1964.

5. Flanders WD, Rothman KJ. Occupational risk for laryngeal cancer. Am J Public Health 1982;72: $369-372$.

6. Gabriel CE, Jones DG. The importance of chronic laryngitis. J Laryngol Otol. 1960 Jun;74: 349-357.

7. Glanz H, Kleinsasser O. Chronic laryngitis and carcinoma. Arch Otorhinolaryngol. 1976 Feb 8; 212 (1):57-75.

8. Wight R, Paleri V, Arullendran P. Current theories for the development of nonsmoking and nondrinking laryngeal carcinoma. Curr Opin Otolaryngol Head Neck Surg. 2003 Apr; 11(2):73-77.

9. Geterud A, Bove M, Ruth M. Hypopharyngeal acid exposure: an independent risk factor for laryngeal cancer? Laryngoscope. 2003 Dec; 113(12):2201-2205.

10. El-Serag HB, Hepworth EJ, Lee P, Sonnenberg A. Gastroesophageal reflux disease is a risk factor for laryngeal and pharyngeal cancer. Am J Gastroenterol. $2001 \mathrm{Jul} ;$ 96(7):2013-2018.

11. Vaezi MF, Hicks DM, Abelson TI, Richter JE. Laryngeal signs and symptoms and gastroesophageal reflux disease (GERD): a critical assessment of cause and effect association. Clin Gastroenterol Hepatol 2003; 1: 333-344.

12. Lacy BE, Weiser K, Chertoff J, Fass R, Pandolfino JE, Richter JE, Rothstein RI, Spangler C, Vaezi MF. The diagnosis of gastroesophageal reflux disease. Am J Med 2010; 123: 583-592.

13. Giannini EG, Zentilin P, Dulbecco P, Vigneri S, Scarlata P, Savarino V. Management strategy for patients with gastroesophageal reflux disease: a comparison between empirical treatment with esomeprazole and endoscopy-oriented ttreatment Am J Gastroenterol 2008; 103: 267-275.

14. Aanen MC, Weusten BL, Numans ME, de Wit NJ, Baron A, Smout AJ. Diagnostic value of the proton pump inhibitor test for gastro-oesophageal reflux disease in primary care. Aliment Pharmacol Ther 2006; 24: 1377-1384.

15. Zentilin P, Dulbecco P, Savarino E, Giannini E, Savarino V. Combined multichannel intraluminal impedance and pHmetry: a novel technique to improve detection of gastrooesophageal reflux literature review. Dig Liver Dis 2004; 36: 565-569

16. Kessing BF, Smout AJ, Bredenoord AJ. Clinical applications of esophageal impedance monitoring and high-resolution manometry. Curr Gastroenterol Rep 2012; 14: 197-205

17. Savarino E, Marabotto E, Zentilin P, Frazzoni M, Sammito G, Bonfanti D, Sconfienza L, Assandri L, Gemignani L, Malesci A, Savarino V. The added value of impedance-pH monitoring to Rome III criteria in distinguishing functional heartburn from non-erosive reflux disease. Dig Liver Dis 2011; 43: 542-547

18. Yan-Junm Wang, Xiu-Qiong Lang, Dan Wu, YuQin He, Chun-Hui Lan, Xiao Xiao, Bin Wang, Duo-Wu Zou, Ji-Min Wu, Yong-Bin Zhao, Peter W Dettmar, Dong-Feng Chen and Min Yang, Salivary Pepsin as an Intrinsic Marker for Diagnosis of Subtypes of Gastroesophageal Reflux Disease and Gastroesophageal Reflux Disease-related Disorders, Journal of Neurogastroenterology and Motility, 10.5056/jnm19032, 26, 1, (74-84), (2020)

19. J. Galli, D. Meucci, G. Salonna, R. Anzivino, V. Giorgio, M. Trozzi, S. Settimi, M.L. Tropiano, G. Paludetti and S. Bottero, Use OF NBI for the assessment of clinical signs of rhino-pharyngo-laryngeal reflux in pediatric age: Preliminary results, International Journal of Pediatric Otorhinolaryngology, 10.1016/j. ijporl.2019.109733 
20. Jian-jun Ren, Yu Zhao, Jing Wang, Xue Ren, Yang Xu, Wenlong Tang and Zhaoping He, PepsinA as a Marker of Laryngopharyngeal Reflux Detected in Chronic Rhinosinusitis Patients, Otolaryngology-Head and Neck Surgery, 10.1177/0194599817697055, 156, 5, (893-900), (2017).

21. Blaser M J. Ecology of Helicobacter pylori in the human stomach. J Clin Investig. 1997;100: 759-762.

22. Hsin-Ching Lin MD, presenter and Chih-Ying Su MD: Helicobacter pylori in the adenoid of children with and without otitis media with effusion Otolaryngology Head and Neck Surgery Volume 131, Issue 2, August 2004, Page P250.

23. Blot WJ, Devesa SS, Kneller RW, et al. Rising incidence of adenocarcinoma of the esophagus and gastric cardia. JAMA 1991;265: 1287 - 1289.

24. Mork J, Glattre E. Squamous cell carcinomas of the head and neck in Norway, 1953-92: an epidemiologic study of a low-risk population. Cancer Causes Control 1998;9: 37 - 48 .

25. Devesa SS, Silverman DT, Young Jr JL, et al. Cancer incidence and mortality trends among whites in the United States, 1947-84. J Natl Cancer Inst 1987;79: 701- 770 .

26. Centers for Disease Control and Prevention. Tobacco use-United States, 1900-1999. MMWR Morb Mortal Wkly Rep 1999;48: 986- 993[erratum in: MMWR Morb Mortal Wkly Rep 48 (1999) 1027].

27. Koufman JA. The otolaryngologic manifestations of gastroesophageal reflux disease: a clinical investigation of 225 patients using ambulatory 24hour $\mathrm{pH}$ monitoring and an experimental investigation of the role of acid and pepsin in the development of laryngeal injury. Laryngoscope 1991;101(53):1 - 78.

28. Olson NR. Effects of stomach acid on the larynx. Proc Am Laryngol Assoc 1983;104: 108 - 112.

29. Morrison MD. Is chronic gastroesophageal reflux a causative factor in glottic carcinoma? Otolaryngol Head Neck Surg 1988; 99:370 - 373.

30. Ward PH, Hanson DG. Reflux as an etiological factor of carcinoma of the laryngopharynx. Laryngoscope 1988;98: 1195- 1199 .

31. Price JC, Jansen CJ, Johns ME. Esophageal reflux and secondary malignant neoplasia at laryngoesophagectomy. Arch Otolaryngol Head Neck Surg 1990; 116: 163 - 164.
32. Frejie JE, Beatty TW, Campbell BH, et al. Carcinoma of the larynx in patients with gastroesophageal reflux. Am J Otolaryngol 1996;17: 386- 390.

33. Biacabe B, Gleich LL, Laccourreye O, et al. Silent gastroesophageal reflux disease in patients with pharyngolaryngeal cancer: further results. Head Neck 1998;510- 514

34. Copper MP, Smit CF, Stanojcic LD, et al. High incidence of laryngopharyngeal reflux in patients with head and neck cancer. Laryngoscope 2000;110:10071011.

35. Lewin JS, Gillenwater AM, Garrett JD, et al. Characterization of laryngopharyngeal reflux in patients with premalignant or early carcinomas of the larynx. Cancer 2003;97: 1010-1014.

36. Galli J, Cammarota G, Calo L, et al. The role of acid and alkaline reflux in laryngeal squamous cell carcinoma. Laryngoscope 2002;112: 1861- 1865.

37. Mercante G, Bacciu T, Ferri T, et al. Gastroesophageal reflux as a possible co-promoting factor in the development of the squamous cell carcinoma of the oral cavity, of the larynx and of the pharynx. Acta Otorhinolaryngol Belg 2003; 57: 113- 7.

38. Bacciu A, Mercante G, Ingegnoli A, Ferri T, Muzzetto $\mathrm{P}$, Leandro $\mathrm{G}$, et al. Effects of gastroesophageal reflux disease in laryngeal carcinoma. Clin Otolaryngol Allied Sci. 2004 Oct; 29(5):545-548.

39. Ward PH, Hanson DG. Reflux as an etiological factor of carcinoma of the laryngopharynx. Laryngoscope 1988; 98: 1195- 1159 .

40. Adhami T, Goldblum JR, Richter JE, et al. The role of gastric and duodenal agents in laryngeal injury: an experimental canine model. Am J Gastroenterol 2004; 99: 2098- 2106.

41. Johnston N, Dettmar PW, Bishwokarma B, Lively MO, Koufman JA. Activity/stability of human pepsin: implications for reflux attributed laryngeal disease. Laryngoscope 2007; 117: 1036-1039.

42. Samuels TL, Johnston N. Pepsin as a marker of extra esophageal reflux. Ann Otol Rhinol Laryngol 2010; 119: 203-208

43. Bardhan KD, Strugala V, Dettmar PW. Reflux revisited: advancing the role of pepsin. Int $\mathrm{J}$ Otolaryngol. 2012;2012 doi: 10.1155/2012/646901. 646901. 
44. Lee AL, Button BM, Denehy L, et al. Exhaled breath condensate pepsin: potential noninvasive test for gastroesophageal reflux in COPD and bronchiectasis. Respir Care. 2015; 60:244-250. doi: 10.4187/ respcare. 03570.

45. Geterude A, Bove M, Ruth M. Hypopharyngeal acid exposure: an independent risk factor for laryngeal cancer? Laryngoscope 2003; 113:2201- 2205.

46. Chen MY, Ott DJ, Casolo BJ, et al. Correlation of laryngeal and pharyngeal carcinomas and 24-hour $\mathrm{pH}$ monitoring of the esophagus and pharynx. Otolaryngol Head Neck Surg 1998; 119: 460 - 2 [retraction in Chen MY, Ott DJ, Koufman JA. Otolaryngol Head Neck Surg 1999; 121:168].

47. Ozlugedik S, Yorulmaz I, Gokcan K. Is laryngopharyngeal reflux an important risk factor in the development of laryngeal carcinoma? Eur Arch Otorhinolaryngol. 2006 Apr; 263(4):339-343.

48. Lundell LR, Dent J, Bennett JR, Blum AL, Armstrong D, Galmiche JP, Johnson F, Hongo M, Richter JE,
Spechler SJ, Tytgat GN, Wallin L. Endoscopic assessment of oesophagitis: clinical and functional correlates and further validation of the Los Angeles classification. Gut 1999; 45: 172-180

49. Ang D, Ang TL, Teo EK, Hsu PP, Tee A, Poh CH, Tan J, Ong J, Fock KM. Is impedance $\mathrm{pH}$ monitoring superior to the conventional 24-h pH meter in the evaluation of patients with laryngorespiratory symptoms suspected to be due to gastroesophageal reflux disease? J Dig Dis 2011; 12: 341-348.

50. Yadlapati R, Adkins C, Jaiyeola DM, et al. Abilities of oropharyngeal $\mathrm{pH}$ tests and salivary pepsin analysis to discriminate between asymptomatic volunteers and subjects with symptoms of laryngeal irritation. Clin Gastroenterol Hepatol. 2016; 14:535-542.e2.

51. Beltsis A, Katsinelos P, Kountouras J, et al. Double probe $\mathrm{pHmonitoring} \mathrm{findings} \mathrm{in} \mathrm{patients} \mathrm{with} \mathrm{benign}$ lesions of the true vocal folds: comparison with typical GERD and the effect of smoking. Eur Arch Otorhinolaryngol. 2011; 268: 1169-1174. 